\title{
Schön und bedroht: Schmetterlinge im Rhein-Main-Gebiet
}

\author{
Christian Offer
}

\begin{abstract}
The Rhein-Main area hosts more than 100 butterfly species, many of which became increasingly endangered and rare. This paper describes some of the native butterfly species. In addition, it provides information on how to protect them by offering habitats in private gardens.

\section{Zusammenfassung}

Im Rhein-Main-Gebiet gibt es über 100 Tagfalter-Arten, doch leider werden es immer weniger. Im Beitrag werden einige Vertreter dieser schillernd-bunten Geschöpfe vorgestellt. Zudem werden Anregungen gegeben, was jeder Einzelne tun kann, damit wir auch in Zukunft Schmetterlinge in freier Natur erleben können.
\end{abstract}

\section{Wunderwelt der Schmetterlinge}

Schmetterlinge sind erstaunliche Geschöpfe. Sie haben Rüssel, die oft länger als ihr ganzer Körper sind. Sie riechen mit ihren Fühlern, schmecken mit den Beinen und tasten mit verschiedenen Stellen ihres Körpers. Ihre Farbenvielfalt begeistert, ihr Flug ist Sinnbild für Leichtigkeit und sommerliche Lebensfreude. Doch viele Schmetterlinge sind vom Aussterben bedroht, schon $80 \%$ der Falter stehen in Deutschland auf der Roten Liste der bedrohten Arten. Dutzende ehemals heimischer Schmetterlings-Arten sind bereits ausgestorben.

Von den etwa 3700 Schmetterlings-Arten in Deutschland zählt der größte Teil zu den so genannten Nachtfaltern. In der Umgangssprache werden Nachtfalter meistens als Motten bezeichnet, jedoch bezieht sich dieser Überbegriff eigentlich nur auf einige kleinere Falter. Andere Nachtfalter-Familien tragen so bezeichnende Namen wie Eulen, Glucken, Schwärmer, Spinner oder Spanner.

Für die meisten Menschen gelten Tagfalter als die eigentlichen Schmetterlinge. Mit ihren prächtigen Farben gehören sie zu einem schönen Sommertag, sei es ein Tagpfauenauge im eigenen Garten, ein Kleiner Fuchs am Wegesrand oder ein imposanter Schwalbenschwanz auf einer Blumenwiese. Die Mehrheit der heimischen Tagfalter ist den Bewohnern der Rhein-MainRegion weitgehend unbekannt. Dies liegt nicht

Abb. 1: Ein Dickkopffalter saugt Nektar aus der Blüte einer Heidenelke, einer typischen Schmetterlingsblume. zuletzt daran, dass viele Vertreter der Schmetterlingsfauna inzwischen sehr selten geworden sind.

\section{Bedrohung der Schmetterlingsvielfalt}

Die Situation der Schmetterlinge im RheinMain-Gebiet ist Besorgnis erregend. Die Gründe dafür sind auch von anderen Arten bekannt: Schuld daran sind wir selbst, weil wir kaum Wildnis zulassen, Wiesen immer früher mähen, Lebensräume beeinträchtigen und zu viele Schadstoffe in die Luft entlassen. Gab es vormals viele nährstoffarme, artenreiche Wiesen, dominieren auf Grünland heute reine Löwenzahn-Bestände, die nur wenige Falter-Arten anziehen. Viele Schmetterlings-Arten haben aber sehr spezielle Ansprüche an ihre Umwelt und sind auf eine

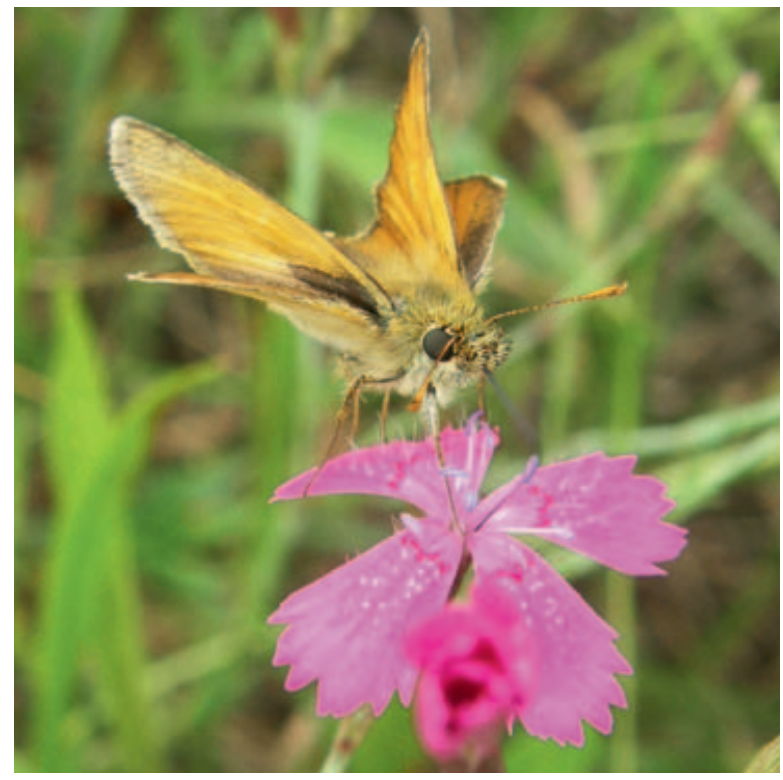



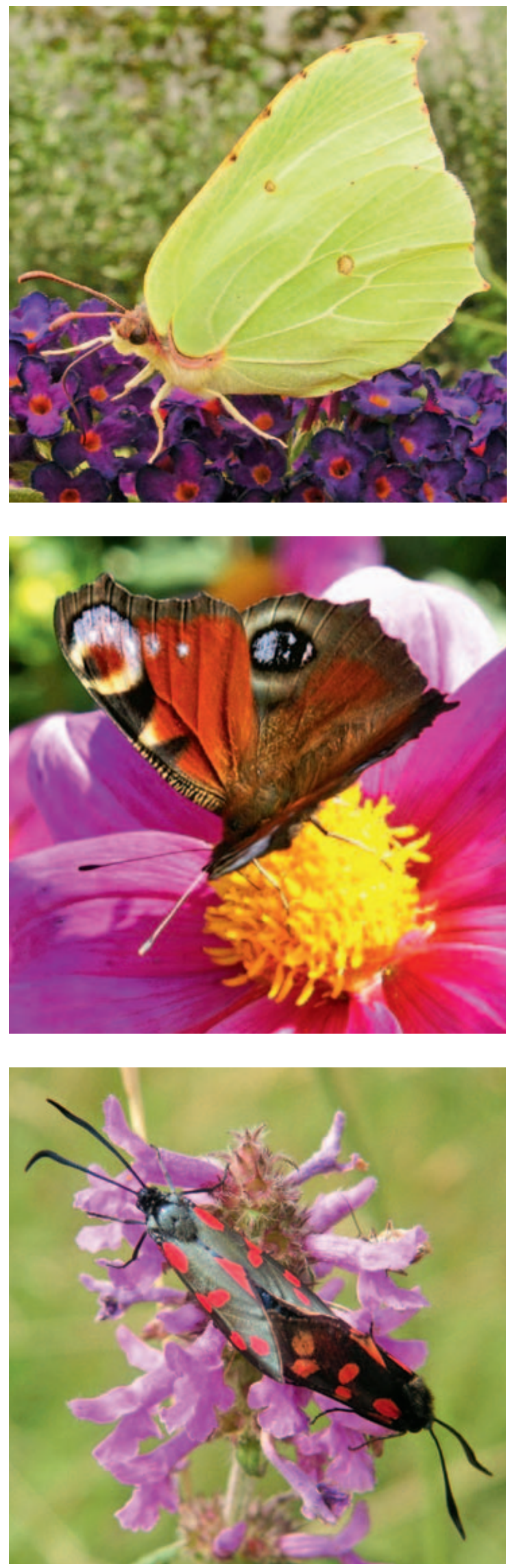

artenreiche Vegetation angewiesen. Die schlüpfenden Raupen benötigen bestimmte Pflanzen und die erwachsenen Falter nutzen oft andere Lebensräume zur Nahrungssuche. Schmetterlinge gelten daher als hervorragende Zeiger-Arten für intakte Lebensräume.

\section{Was kann man persönlich zum Erhalt der Schmetterlingsvielfalt tun?}

Schmetterlingen im eigenen Garten zu helfen ist gar nicht so schwer. Ein durchgestalteter Garten ist allerdings für Schmetterlinge ungeeignet. Mit etwas Mut zur Wildnis und zum Nichtstun ist der erste Schritt getan. Für Tagpfauenaugen und viele andere SchmetterlingsArten sind Brennnessel-Ecken lebensnotwendig, da ihre Raupen auf die Brennnessel als Futterpflanze angewiesen sind. Neben Brennnesseln sind unter anderem viele Gräser, Disteln und andere Korbblütler, aber auch Doldenblütler wie Fenchel, Möhre und Dill wichtige Futterpflanzen für Schmetterlings-Raupen. In einem Garten sollte deshalb nicht überall ein „steriler Golfrasen“ gepflegt werden, eine bunte Blumenwiese mögen Schmetterlinge lieber.

Schmetterlinge anzulocken heißt aber auch nicht, dass man unbedingt alles verwildern lassen muss. Ein bestimmter Bereich im Garten reicht dafür schon aus. Erwachsene Schmetterlinge sind nicht besonders wählerisch bei der Futtersuche. Wichtig ist, dass die Blüten reichlich Nektar führen, der für Schmetterlinge gut zugänglich ist. Großblumige einjährige Pflanzen, Stauden und nicht überzüchtete Blumen sind willkommen. Achten Sie darauf, möglichst nur einheimische Pflanzen zu verwenden. Ob Admiral, Distelfalter, Schachbrett oder sogar der prächtige Schwalbenschwanz, sie alle und noch viele mehr werden die $(G)$ Artenvielfalt mit ihrer Anwesenheit belohnen.

Abb. 2 (oben): Der Schmetterlingsflieder bietet nicht nur Zitronenfaltern reichlich Nahrung.

Abb. 3 (Mitte): Tagpfauenauge, einer unserer auffälligsten und noch relativ häufigen Falter.

Abb. 4 (unten): Krainer Widderchen bei der Paarung. 


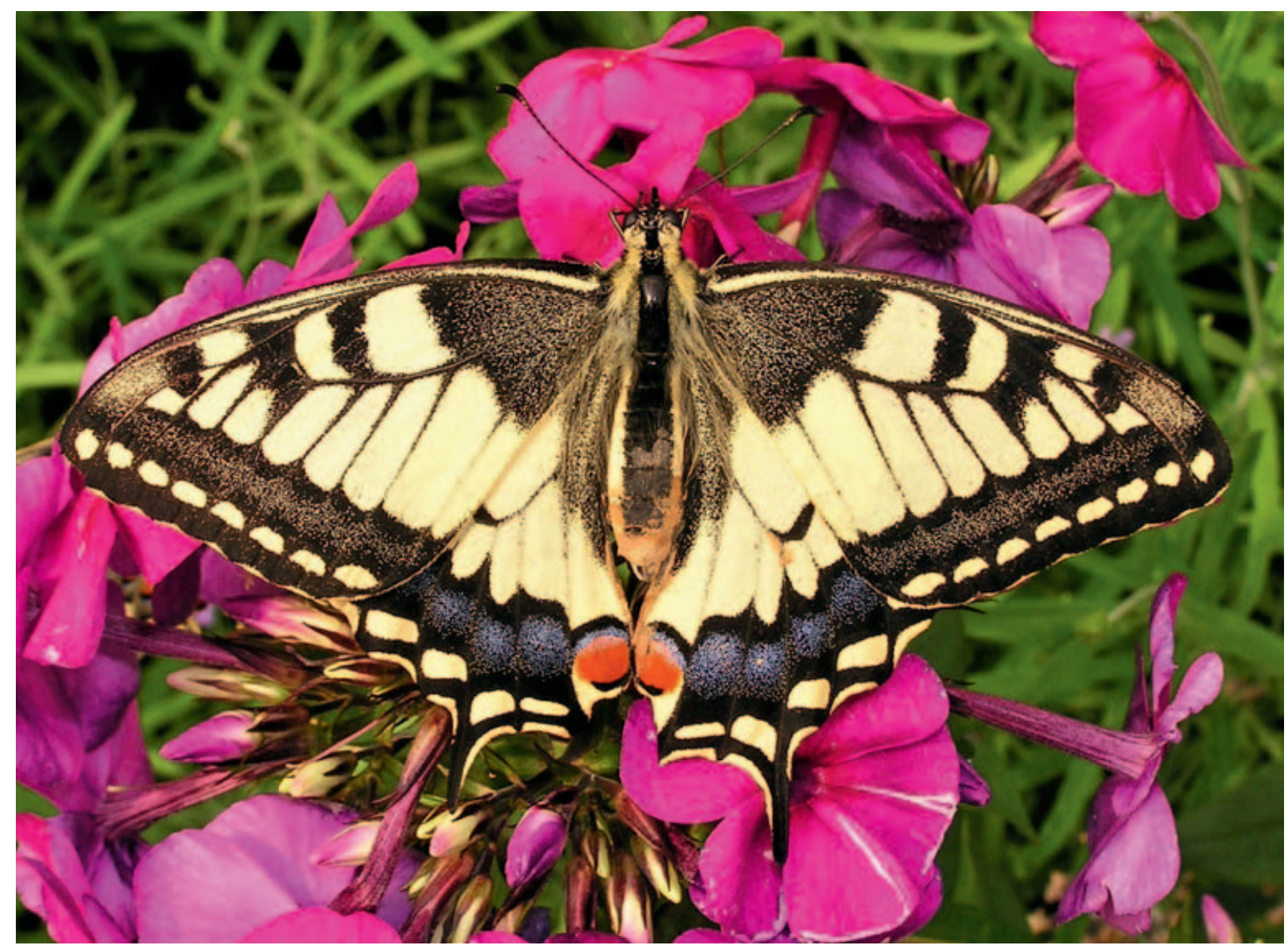

\section{Bestandsaufnahme von Schmetterlingen - helfen Sie mit}

Mit ein bisschen Einarbeitung kann jeder für den Schutz der heimischen SchmetterlingsArten aktiv werden. Besorgen Sie sich einschlägige Bestimmungsliteratur (siehe Literaturliste) und lesen Sie sich etwas ein. Wenden Sie sich an das Umweltforschungszentrum (UFZ) LeipzigHalle unter www.tagfalter-monitoring.de, tagfalter-monitoring@ufz.de. Das UFZ koordiniert das so genannte Tagfalter-Monitoring bundesweit. Geschulte Laien und Experten können unter Anleitung in die Natur ausschwärmen und so viele Schmetterlinge zählen wie möglich. Die Aufnahmebögen mit ausführlicher Anleitung können Sie per E-Mail über den Autor bestellen.

Abb. 5: Schwalbenschwanz.

\section{Auffällige Schmetterlinge im Rhein-Main-Gebiet}

5.1 Das Krainer Widderchen (Zygaena carniolica), Insekt des Jahres 2008

Seinen Namen verdankt der kleine Falter seinen gebogenen Fühlern. Mit seinen schwarz umrahmten roten Flecken sieht es blutig gefleckt aus, weshalb der Volksmund es wie auch 12 weitere Widderchen-Arten Blutströpfchen nennt. Die Flecken signalisieren: Friss mich nicht - ich bin giftig. Widderchen lieben die mageren Wiesen der Grünlandflächen im Rhein-MainGebiet, die leider zunehmend Straßen und Siedlungen weichen. Vor allem an Südhängen und auf Streuobstwiesen des Hochtaunus fühlen sich die kleinen Falter wohl. Schon vor 30 Mio. Jahren existierten die Blutströpfchen-Falter. Um auf ihre Gefährdung aufmerksam zu machen, erhielt das Krainer Widderchen stellvertretend für alle anderen Blutströpfchen-Arten die Auszeichnung als „Jahresinsekt". 

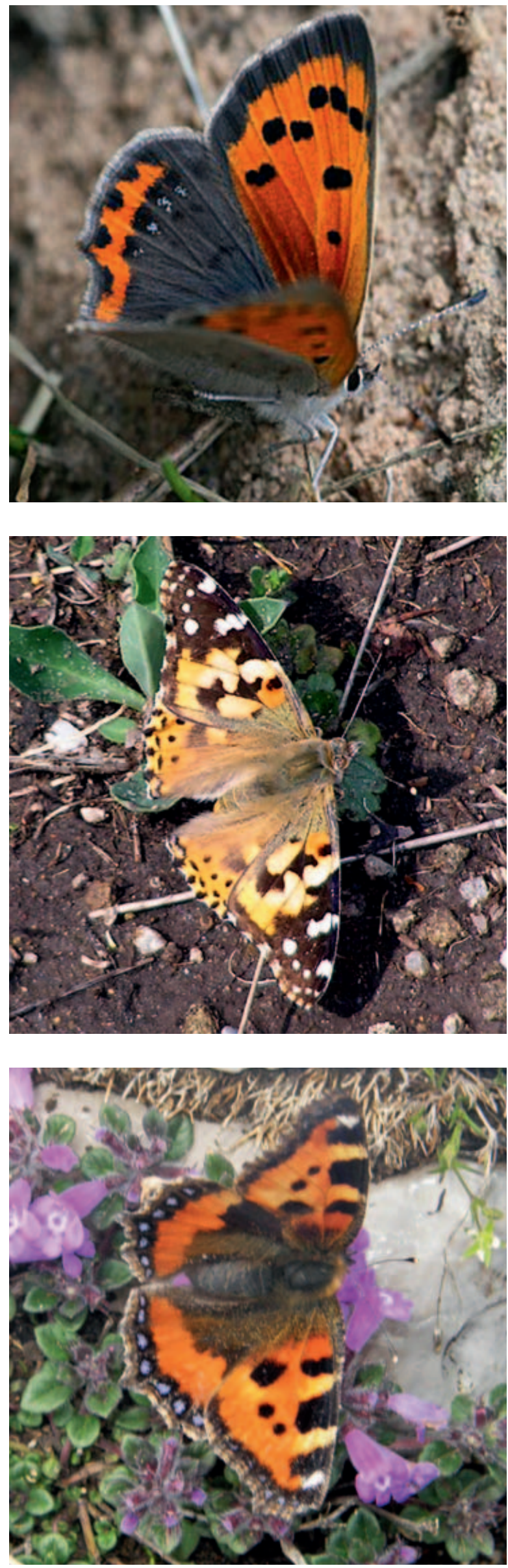

Die folgenden Artenporträts stammen von der Aktionsgemeinschaft „Rettet den Burgwald e.V.“

\subsection{Schwalbenschwanz (Papilio machaon)}

Der Schwalbenschwanz ist mit seiner charakteristischen gelb-schwarzen Flügelzeichnung vielleicht der schönste, sicherlich aber einer der größten heimischen Tagfalter. Mitunter ist er schon im April bei uns zu entdecken. Bis zum September bildet er zwei bis drei Generationen aus. Die weit umherschweifenden Falter bevorzugen als Lebensraum Mager- und Trockenrasen, Brachflächen, Streuobstwiesen, Gemüsegärten und nicht überdüngte Mähwiesen mit hohen Anteilen an Doldengewächsen, welche die Nahrungspflanzen der Raupen darstellen. Dazu zählen die Wilde und die Garten-Möhre, Fenchel, Dill, Haarstrang-Arten, Petersilie oder Kleine Bibernelle.

In der Roten Liste der Tagfalter Hessens wird der Schwalbenschwanz als eine Art der Vorwarnliste (V) geführt, d.h. sein Bestand ist in den letzten Jahren merklich zurückgegangen. Ursachen dafür sind die Überdüngung und intensive Nutzung von Grünland, die Anwendung von Pflanzenschutzmitteln, die häufige Mahd von Wegrändern und Böschungen sowie das Mulchen von Brachflächen und Gemüsefeldern. Beim Fortbestehen dieser schädigenden Einflüsse ist ein weiterer Rückgang dieser Art wahrscheinlich.

\subsection{Kleiner Feuerfalter (Lycaena pblaeas)}

Der Kleine Feuerfalter gehört noch zu den weit verbreiteten Tagfaltern. Dabei wird dieser zur Familie der Bläulinge zählende, relativ kleine und auf den ersten Blick unscheinbar wirkende Falter häufig übersehen. Nachdem seine an Ampfer-Arten lebenden Raupen den Winter überstanden haben, kann man den Kleinen Feuerfalter ab April bei uns finden. Er zeigt sich bis in den Oktober hinein (auch häufig in Gärten) und hat innerhalb dieser Zeit vier, an opti-

Abb. 6 (oben): Kleiner Feuerfalter.

Abb. 7 (Mitte): Distelfalter.

Abb. 8 (unten): Kleiner Fuchs. 
malen Standorten möglicherweise auch fünf Generationen gebildet, die sich stark überschneiden. Die Individuenzahl nimmt dabei im Normalfall mit den Generationen zu, so dass im Spätsommer mit den meisten Faltern zu rechnen ist.

\subsection{Distelfalter (Vanessa cardui)}

Der Distelfalter zählt zu den Wanderfaltern und wandert im Frühling und Frühsommer aus dem Süden nach Mitteleuropa. Dabei legt er einen weiten Weg zurück, denn seine Ursprungsgebiete liegen im nördlichen Afrika und sogar südlich der Sahara. Zu uns einwandernde Falter stammen aus Afrika oder Süd-Europa. Offenbar schon im Hochsommer begeben sich dann die ersten bei uns herangewachsenen Falter wieder auf den Rückweg in den Süden. Als Futterpflanzen für die Raupen dienen nicht nur diverse Distel-Arten, sondern z. B. auch Brennnessel, Malven, Wegerich und Klette.

\subsection{Kleiner Fuchs (Aglais urticae)}

Der Kleine Fuchs zählt zu den bekanntesten und am weitesten verbreiteten heimischen Tagfaltern. Er fliegt sowohl im offenen Gelände als auch auf Waldlichtungen oder entlang von Forstwegen und Schneisen. Häufig ist er auch in Gärten anzutreffen. In Kellern, auf Dachböden oder in Gartenschuppen finden sich im Winter nicht selten ruhende Falter, die hier die kalte Jahreszeit zu überstehen versuchen. Solche Tiere sollte man nicht stören und auch nicht in wärmere Räumlichkeiten umsiedeln. Diese Überwinterer gehören zu den früh im Jahr erscheinenden Schmetterlingen. Bis zum Oktober entstehen mehrere Generationen, deren Raupen sich von Brennnesseln ernähren.

\subsection{Landkärtchen (Araschnia levana)}

Das Landkärtchen gehört zu den verbreiteten Tagfalterarten, deren Raupen ausschließlich Brennnessel-Blätter fressen. Die beiden sich pro Jahr entwickelnden Falter-Generationen unter-

Abb. 9 (oben): Landkärtchen in der Sommerform.

Abb. 10 (unten): Schachbrettfalter.
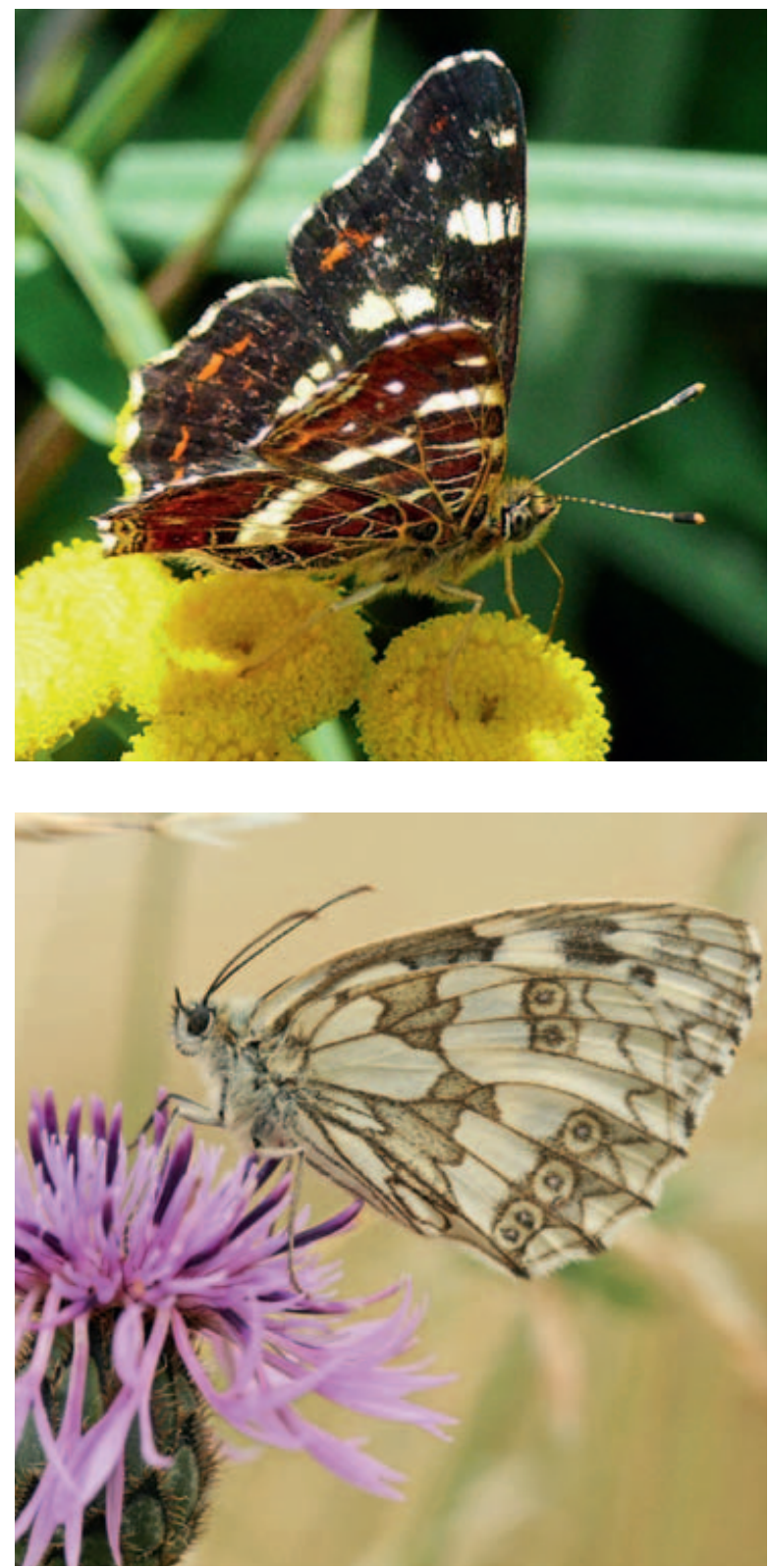

scheiden sich stark. Die Frühlingsgeneration hat eine orange-braune Grundfarbe der FlügelOberseite mit schwarzer Fleckung, die Sommergeneration zeigt dagegen eine schwarze Grundfärbung mit weißen und orangen Bändern. Beide Generationen tragen auf den Flügel-Unterseiten eine Zeichnung aus feinen Linien, Bändern und Flecken. Diese soll an eine Landkarte erinnern und gab dem Falter seinen deutschen Namen.

\subsection{Schachbrettfalter (Melanargia galathea)}

Das Schachbrett ist einer der auffälligsten Sommerfalter und findet sich vorwiegend auf wenig gedüngtem, blumenreichen Gras- und Offen- 


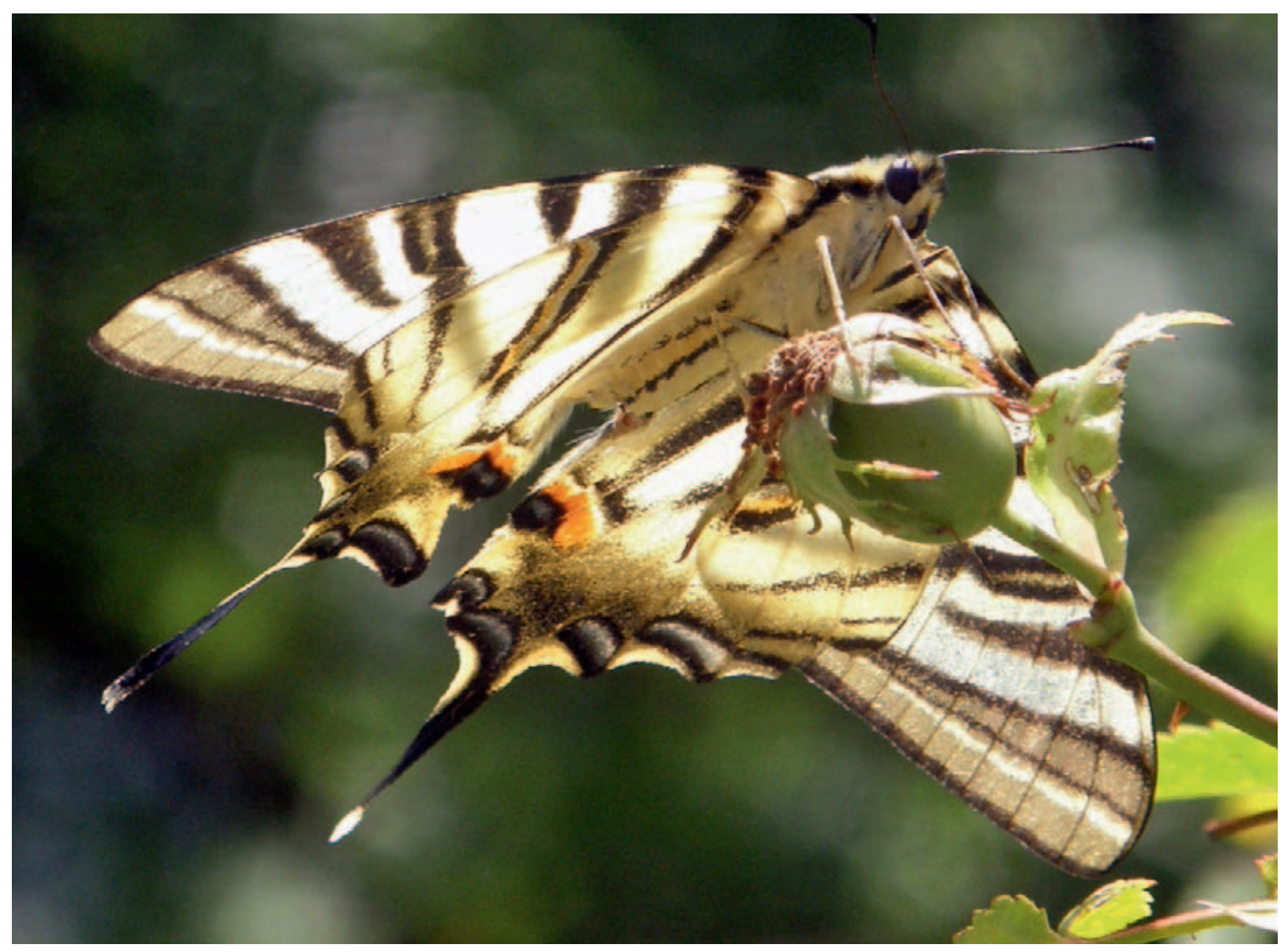

land, ist aber auch entlang von Gebüschrändern und Waldwegen anzutreffen. Die intensiv betriebene Landwirtschaft und die Pflege auch von ungenutzten Grünflächen haben auch diese Art inzwischen deutlich dezimiert. Die weiblichen Falter legen ihre Eier bevorzugt in ungemähten Grasbeständen ab, die sich entwickelnden Raupen leben an verschiedenen Gräsern und überwintern als Jungraupe.

\section{Dank}

Herzlicher Dank gilt Dr. Wolfgang Nässıg für die kritische Durchsicht des Manuskriptes sowie der Aktionsgemeinschaft „Rettet den Burgwald e.V.“ für die Überlassung von Bildmaterial sowie der Artenportraits 5.2-5.7.

\section{Literatur}

Bellmann, H. 2003: Kosmos-Schmetterlingsführer. Stuttgart.

DiERL, W. 2005: BLV Naturführer Schmetterlinge. München.
WitT, R. 2001: Ein Garten für SchmetterlingeStuttgart.

Eine umfangreiche Publikation über Schmetterlinge hat der BUND herausgegeben. Sie erhalten sie auf Anfrage bei BioFrankfurt (Christian Offer, Tel.: 06979824757, E-Mail: offer@biofrankfurt.de).

\section{Auswahl weiterführende Internetseiten}

www.abenteuer-schmetterling.de www.ag-burgwald.de/?Fauna:Schmetterlinge www.biofrankfurt.de www.mein-schoener-garten.de/jforum/posts/ list/1402800.page www.Schmetterlinge-Deutschlands.de www.schmetterling-raupe.de

\section{Schmetterlingsbestimmung für den Naturschutz:} www.lepiforum.de/cgi-bin/bestimmung.pl www.science4you.org/platform www.tagfalter-monitoring.de

Abb. 11: Segelfalter, Beispiel für eine nur noch selten anzutreffende Schmetterlings-Art. Um ihn zu erhalten, muss unbedingt auch sein Lebensraum geschützt werden. 